\title{
Application of Mössbauer Spectroscopy in Study of Selected Biochemical Processes
}

\author{
K. BURDA ${ }^{a}$ AND J. STANEK ${ }^{b, *}$ \\ ${ }^{a} \mathrm{H}$. Niewodniczanski Institute of Nuclear Physics \\ Radzikowskiego 152, 31-342 Kraków, Poland \\ ${ }^{b}$ M. Smoluchowski Institute of Physics, Jagiellonian University \\ Reymonta 4, 30-059 Kraków, Poland
}

(Received January 15, 2003)

\begin{abstract}
The ${ }^{57} \mathrm{Fe},{ }^{119} \mathrm{Sn},{ }^{129} \mathrm{I}$, and ${ }^{151} \mathrm{Eu}$ Mössbauer spectroscopy, scanning force microscopy, and optical fluorescence method were applied to study biological systems starting from porphyrins, through cytochromes and cell membranes until such a complex system as photosystem II. In Fe-porphyrin aggregates iron atoms are able to trap an electron exhibiting the mixed valence $\mathrm{Fe}^{3+}-\mathrm{Fe}^{2+}$ relaxation process. In ironcytochrome $\mathrm{c}$ the presence of two different $\mathrm{Fe}^{3+}$ states are indicated, while in tincytochrome $\mathrm{Sn}$ appears in $\mathrm{Sn}^{4+}$ and $\mathrm{Sn}^{2+}$ states. From the temperature dependence of the mean square displacement of the resonance nuclei and from the diffusional broadening of the Mössbaner line it was possible to separate the vibrational, fast collective and slow collective motions in tinporphyrin and in iron- and tin-cytochrome c. The electronic state of iodine in oleic acid, the main constituent of cellular membranes, was determined. The molecular mechanism of triphenyltin interaction with membrane of red blood cells has been suggested and the model of haemolysis has been proposed. In photosystem II, Eu ions replacing calcium showed $\mathrm{Eu}^{3+}$ to $\mathrm{Eu}^{2+}$ transition after illumination with light, which points out the possible role of $\mathrm{Ca}^{2+}$ ions in electron transfer in the process of photosynthetic water splitting process.
\end{abstract}

PACS numbers: 82.80.Ej, 82.39.Jn, 82.35.Np, 82.39.Wj, 82.39.Rt

*corresponding author; e-mail: jstanek@theta.uoks.uj.edu.pl 


\section{Introduction}

Mössbauer spectroscopy, a local nuclear method, is known to be a powerful method in the determination of electronic, structural, and dynamical properties of certain isotopes in solids. It turns out that the applicability of this method can be extended to biological complexes providing information on electron and energy transfers in living systems. Some Mössbauer probes play a crucial role in life processes. The example of iron is here the most spectacular one.

The study of the valence and spin state of metals in porphyrins, the active biologically centers, is important for the general understanding of the metabolic function of a protein which is based on modification of its redox potential. Ironporphyrin (haem group) has been found in many complexes, which are important for living processes like haemoglobin, myoglobin (the carriers of oxygen) or cytochromes (the electron transporting proteins) [1]. In particular, cytochrome $\mathrm{c}$ is a component of all organisms having mitochondrial breath chain [2]. Its redox potential $(+0.25 \mathrm{~V})$ and its tertiary structure are highly conserved. For example, the axial haem ligands: Met80 and His18 as well, the two cysteins covalently binding the haem moiety, remain unchanged in about 30 cytochromes $c$ originated from various species. Haem is a redox active center of cytochrome c. The valence and spin states of iron as well as the flexibility of this protein are crucial for its activity. However, action of redox active enzymes is additionally regulated by the rigidity of biomembranes. Generally, cellular metabolism is strongly correlated to the fluidity of the membrane depending on the proportion between saturated and unsaturated fatty acids [3] and on the structure of cytoskeleton [4].

In this paper we give a brief review of some applications of Mössbauer spectroscopy in biological systems based on our own results. At first, we discuss the valence and spin states of iron and tin in porphyrin IX and cytochrome c substituted with ${ }^{57} \mathrm{Fe}$ and ${ }^{119} \mathrm{Sn}$ ions. We compare the flexibility of these two ions in the different matrices. Then, we present the Mössbauer investigations of ${ }^{129} \mathrm{I}$ bound to oleic acid, which is a constituent of biomembranes, and to its saponificated form. The rigidity of membranes could be studied using iodine anions saturating $\mathrm{C}=\mathrm{C}$ bonds in unsaturated acids. We show a highly specific interaction of triphenyltin chloride with the membrane fragments of red blood cells, being a model system. This proposed mechanism of the interaction of tin with cytoskeleton explains high toxicity of this compound which influences the transport processes through biological membranes in an early phase of the lysis of poisoned cells. Finally, we show new data concerning oxygen evolving complex in photosystem II in which $\mathrm{Ca}^{2+}$ ions have been replaced by $\mathrm{Eu}^{3+}$. One of the most intriguing problem in the process of photosynthesis is the requirement of such inorganic cofactors as $\mathrm{Cl}^{-}$and $\mathrm{Ca}^{2+}$ ions for the activation of the oxygen evolving complex [5]. Our data supply hints for solving this problem. 


\section{Materials and methods}

The synthesis of Sn and Fe cytochromes c (50\% enrichment in ${ }^{57} \mathrm{Fe}$, natural abundance ${ }^{119} \mathrm{Sn}$ ) has been done according to Vanderkooi [6] and Burnham and Zuckerman [7] with some modifications [8]. The purity of the compounds was checked by the optical fluorescence method.

The method of oleic acid saturation and then its saponification is described in [9]. The radioactive ${ }^{129} \mathrm{I}$ (half lifetime of $1.6 \times 10^{7} \mathrm{y}$ ) used for preparation of the absorbers was purchased from Oak Ridge in the standard form of $\mathrm{NaI}$ $\left(85-99 \%{ }^{129} \mathrm{I}\right)$ in a basic sulfite solution. The occurrence of iodination was checked by infrared Fourier spectroscopy in the range of $700-40000 \mathrm{~cm}^{-1}$.

The treatment of red blood cells with organotins is described in [10]. The haemolytic effect of trimethyltin chloride $\left(\mathrm{Me}_{3} \mathrm{SnCl}\right)$, tri- $n$-propyltin chloride $\left(\mathrm{Pr}_{3} \mathrm{SnCl}\right)$ and triphenyltin chloride $\left(\mathrm{Ph}_{3} \mathrm{SnCl}\right)$ has been detected by measurements of absorbance at $546 \mathrm{~nm}$ in the supernatant by means of SLM Aminco DW2000 spectrophotometer. The elasticity of the intact red blood cells and cells treated with various toxicants has been measured using scanning force microscope (SFM) working in contact mode [11].

Thylakoid membranes enriched in photosystem II (PSII) has been prepared according to Berthold et al. [12]. The procedure of substitution calcium ions by europium ions is given in [13]. The stimulation or inhibition of oxygen evolution by $\mathrm{Eu}^{3+}$ has been measured using "the three electrode system" [14].

The principles of Mössbauer spectroscopy are described in numerous monographs, for example see [15]. The presented here transmission Mössbauer spectra were recorded between 4.2 and $300 \mathrm{~K}$. The studied samples contained natural abundance of ${ }^{119} \mathrm{Sn}$ and ${ }^{151} \mathrm{Eu}$ or were enriched, as indicated above, in ${ }^{57} \mathrm{Fe}$ and ${ }^{129} \mathrm{I}$.

\section{Results and discussion}

\subsection{Local electronic states of the iron and tin ions in porphyrin and cytochrome $c$}

We show the Mössbauer spectra of the studied metalloporphyrins and metallocytochromes $\mathrm{c}$ in Fig. 1 and Fig. 2. In the case of ironporphyrin the spectrum at room temperature consists of two apparent $\mathrm{Fe}^{3+}$ quadrupole doublets related to two distinguished states of iron. One of them is able to trap an electron exhibiting the mixed valence $\mathrm{Fe}^{3+}-\mathrm{Fe}^{2+}$ relaxation process. This is pronounced in spectra detected at lower temperatures where the component of the ferrous state is visible (Fig. 1). A simplified relaxation model $[8,16]$ gives the activation energy of electron trapping of about $10 \mathrm{meV}$. If ironporphyrin is built into cytochrome $\mathrm{c}$ the metal sites are separated and there is no electron transfer. However, the Mössbauer spectra of ironcytochrome $c$ indicate a presence of two different chemical environments of $\mathrm{Fe}^{3+}$ (Fig. 2a), which can originate from a different ligand or a different symmetry of ligation caused by modification of the protein matrix due to the preparation method. In Sn-porphyrin we observe only one $\mathrm{Sn}^{4+}$ state in the whole temperature 


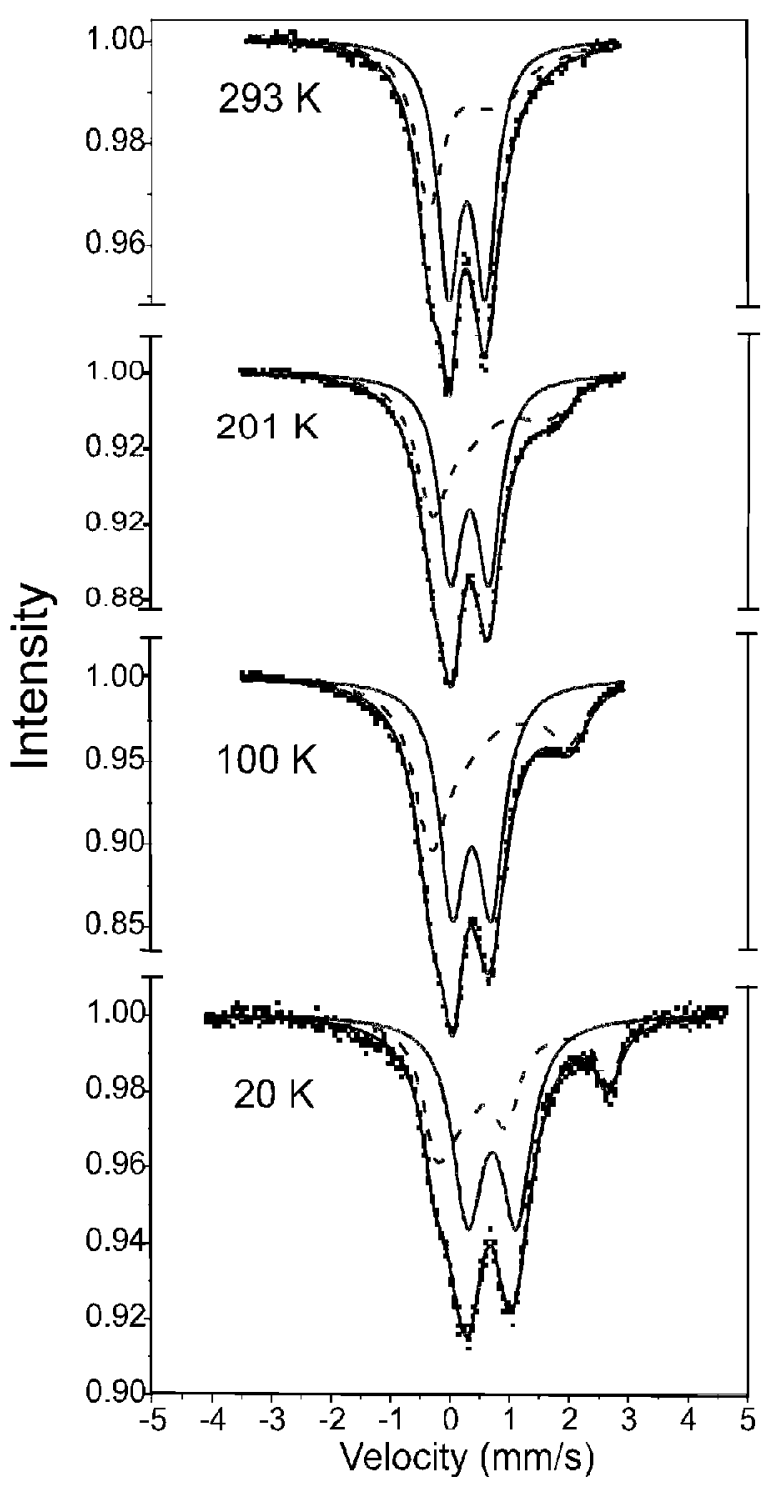

Fig. 1. $\quad{ }^{57}$ Fe Mössbauer spectra of ironporphyrin at temperatures as marked. The spectra consist of the static $\mathrm{Fe}^{3+}$ doublet and the $\mathrm{Fe}^{3+}-\mathrm{Fe}^{2+}$ relaxation spectrum (dashed line).

range (between $78-293 \mathrm{~K}$ ), whereas in the case of tincytochrome $c$ there are two Sn valence states: $\mathrm{Sn}^{4+}$ and $\mathrm{Sn}^{2+}$ (Fig. 2b) [8]. The $\mathrm{Sn}^{2+}$ state in Sn-cytochrome may be related to four-coordinated tin while the $\mathrm{Sn}^{4+}$ states in Sn-porphyrin and Sn-cytochrome are probably related to a six coordinated site with two axial $\mathrm{Cl}^{-}$ ligands as suggested by fluorescence measurements. 

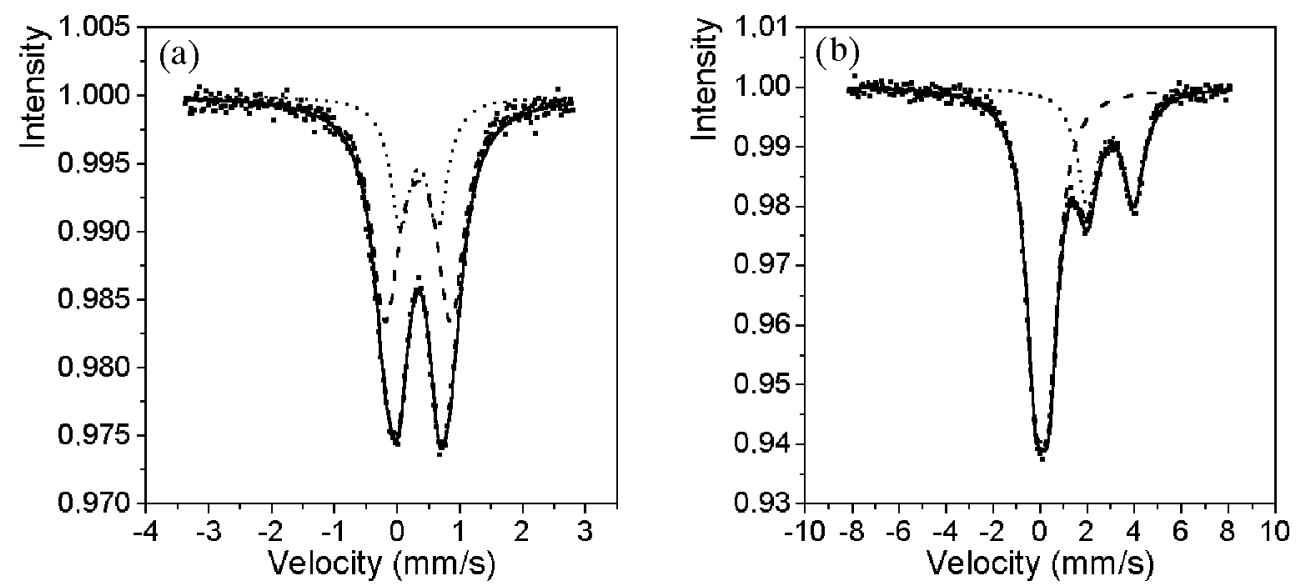

Fig. 2. Mössbauer spectra of metallocytochromes at $\mathrm{LN}_{2}$ temperature: (a) ${ }^{57} \mathrm{Fe}$ spectrum of ironcytochrome c, (b) ${ }^{119} \mathrm{Sn}$ spectrum of tincytochrome c.

\subsection{Dynamics of metalloporphyrins and metallocytochromes}

Biomolecules are usually large flexible systems. Many experimental techniques point to rapid conformational fluctuations of proteins, which play an important role in their physiological activity. Mössbauer spectroscopy can be used as a complementary method in a study of biomolecular dynamics.

As an example we present results for lyophilized iron-, tin-porphyrin and iron-, tin-cytochrome c measured over a wide range of temperatures $(78-340 \mathrm{~K})$. The mean square displacement $\left\langle x^{2}\right\rangle$ of the resonance nucleus $\left({ }^{57} \mathrm{Fe}\right.$ or ${ }^{119} \mathrm{Sn}$ in our case) can be determined from the Lamb-Mössbauer factor: $f=\exp \left(-4 \pi\left\langle x^{2}\right\rangle / \lambda^{2}\right)$, where $\lambda$ is a wavelength of the employed gamma radiation. The total mean square displacement is usually approximated by a sum of three statistically independent components [17]:

$$
\left\langle x^{2}\right\rangle=\left\langle x^{2}\right\rangle_{\mathrm{v}}+\left\langle x^{2}\right\rangle_{\mathrm{fc}}+\left\langle x^{2}\right\rangle_{\mathrm{sc}} .
$$

The subscripts v, fc, sc correspond to vibrational, fast collective, and slow collective motions, respectively. Slow collective motions of characteristic time $10^{-8} \mathrm{~s}$ are associated with bounded diffusion [18]. They lead to the line broadening, which is proportional to the diffusional mean square displacement $\left(\left\langle x^{2}\right\rangle_{\mathrm{sc}}\right)$. This phenomenon, which is common in frozen solution of proteins, we observed in the lyophilized sample of iron-porphyrins above $300 \mathrm{~K}$ (Fig. 3). In the case of lyophilized tin-porphyrin and iron-, tin-cytochrome c, we detected only vibrational and fast collective motions in the studied range of temperatures. Below the critical temperature, when the characteristic time of the conformational relaxation is long relative to the nuclear lifetime, only vibrational motions contribute to $\left\langle x^{2}\right\rangle$. Above the critical temperature, fast fluctuations between different conformational substates occur. They 


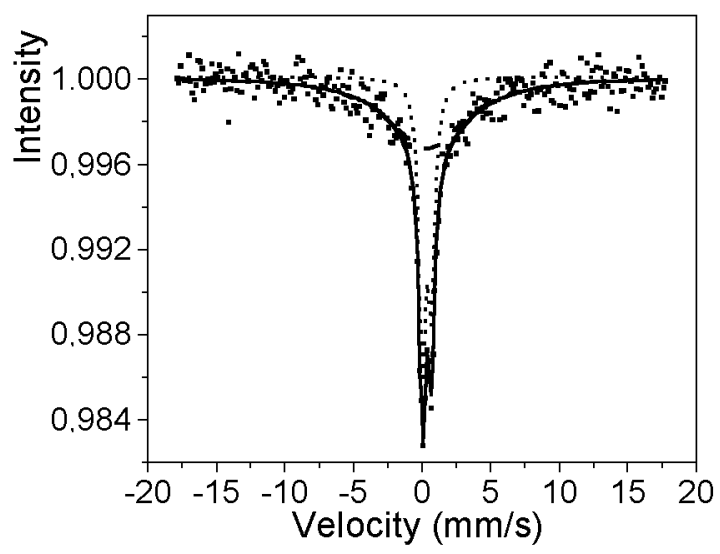

Fig. 3. ${ }^{57} \mathrm{Fe}$ Mössbaner spectrum of lyophilized ironporphyrin recorded above $300 \mathrm{~K}$. The diffusion broadening is visible.

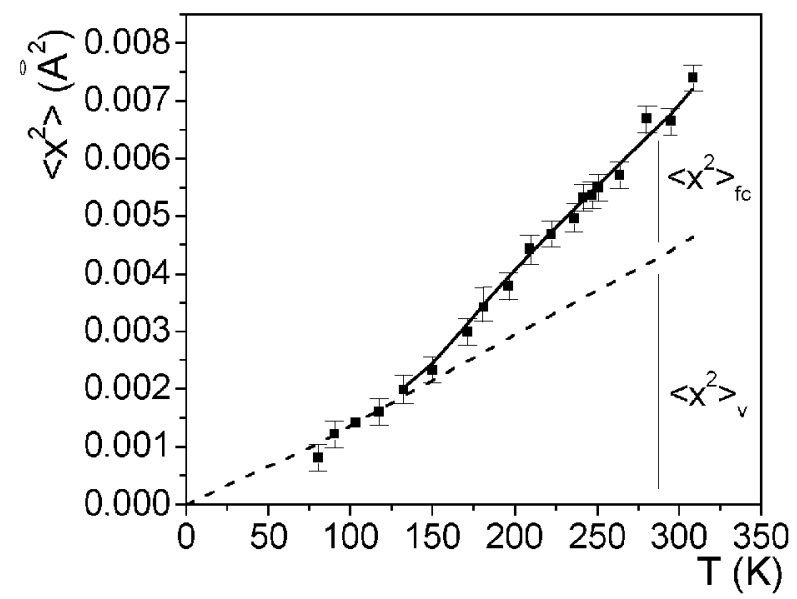

Fig. 4. Temperature dependence of the mean square displacement $\left\langle x^{2}\right\rangle$ of $\mathrm{Sn}$ in tinporphyrin. The contribution from vibrational $\left\langle x^{2}\right\rangle_{\mathrm{v}}$ and fast collective $\left\langle x^{2}\right\rangle_{\mathrm{fc}}$ motions are indicated.

are associated with a steep decrease in the total resonance absorption with increasing temperature. As an example we show in Fig. 4 the temperature dependence of the mean square displacement of the Sn nucleus in Sn-porphyrin IX. The contributions from the vibrational and fast collective motions are indicated. The solid line was fitted according to the double-state model [19, 20] assuming an existence of only two main conformational substates separated by a potential barrier $Q$ and distance $d$. The energy difference between the substates in Fe- and Sn-cytochrome c is the same within the error $(E=78 \mathrm{meV})$, whereas it is about two times smaller for Sn-porphyrin IX $(E=37 \mathrm{meV})$. This indicates a higher symmetry between 
the occupation of the two conformational substates in Sn-porphyrin. The highest energy barrier was observed for $\mathrm{Sn}^{2+}$ in cytochrome c $(Q=202 \pm 5 \mathrm{meV})$ and the lowest value was obtained for $\mathrm{Sn}^{4+}$ in cytochrome c $(Q=118 \pm 5 \mathrm{meV})$. The energy barrier in the case of Fe-cytochrome c $(Q=132 \pm 10 \mathrm{meV})$ is comparable to that observed for $\mathrm{Sn}^{4+}$-porphyrin $(Q=159 \pm 6 \mathrm{meV})$.

The Debye model extended for anharmonicity $[8,20]$, not discussed in this paper, is an alternative approach of describing the decrease in the resonant absorption effect with increasing temperature.

\subsection{Rigidity of cell membranes}

\subsubsection{Possible application for studies of native systems}

Mössbauer spectroscopy can be applied not only for investigation of the dynamic properties of a single molecule but also to study diffusional motion of more complex systems as for example biological membranes, which fluidity is a crucial for a proper cellular metabolism. Fatty acids substituted by halogens (for example ${ }^{129} \mathrm{I}$ ) might be a good probe for such studies. They can be easily built in the biomembranes. The ${ }^{129} \mathrm{I}$ Mössbauer spectrum of iodine in oleic acid is shown in Fig. 5. We have shown that the saponification of the oleic acid substituted by iodine

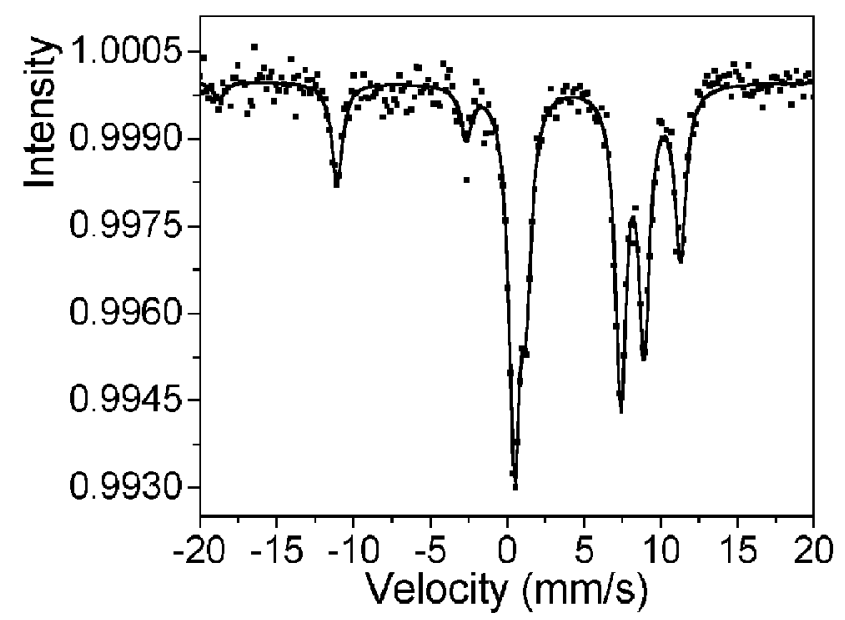

Fig. 5. ${ }^{129} \mathrm{I}$ Mössbauer spectrum of iodine in oleic acid at LHe temperature.

does not influence the electronic configurations of iodine, which have been found to be $5 s^{1.95} 5 p^{5.21}$. The saponification causes only a narrowing of the resonance line because of a higher ordering of the modified system [9]. This permits that temperature changes in the Mössbauer spectrum of iodine should be indicative for the mobility of membranes. Thus, Mössbauer spectroscopy on ${ }^{129} \mathrm{I}$ may be a new tool in studies of the rigidity of membranes. 


\subsubsection{Studies of cell lysis}

Organotins belong to the most common toxicants for living organisms [21, 22]. They are easily soluble in the lipid fraction of cell membranes. Their location and degree of cell disruption depend on the hydrophobicity of the alkyl chain. We found that the haemolytic activity of triphenyltin is similar to tri- $n$-propyltin but much higher in comparison to trimethyltin [10]. This is in agreement with the hydrophilic character of trimethyltin. The Mössbauer studies of lyophilized fractions of supernatants containing haemoglobin and sediments containing erythrocyte membranes in red blood cells treated with triphenyltin $\left(\mathrm{Ph}_{3} \mathrm{SnCl}\right)$ and trimethyltin $\left(\mathrm{Me}_{3} \mathrm{SnCl}\right)$ chloride showed that there is no interaction of the organotins with pig haemoglobin. So far, only cat and rat haemoglobins have been found to bind organotins with a highly specific way $[23,24]$. The $\mathrm{S}_{\text {thiol }}$ cysteine and $\mathrm{N}_{\text {het }}$ histidine were suggested to be ligands of organic compounds of tin in these haemoglobins and there is lack of that histidine residue in the pig haemoglobin. However, we have detected a highly specific interaction of triphenyltin with the erythrocyte membranes. The Mössbauer spectrum in form of a single doublet is characterized by hyperfine parameters that differ considerably from those reported for other organotin compounds in membranes of red blood cells (isomer shift $1.25(1) \mathrm{mm} / \mathrm{s}$ and quadrupole splitting $1.74(1) \mathrm{mm} / \mathrm{s}$ ). Applying the partial quadrupole splitting approach we concluded that ankyrin and $\beta$-spectrin, the cytoskeletal proteins, are the most probable targets of triphenyltin action [10]. They contain highly conserved amino acid sequences providing a similar to the cat (rat) haemoglobin tertiary arrangement of histidine and cysteine residues. Such specific interaction of triphenyltin with these membrane cytoskeleton components influences the rigidity of red blood cells already at the preliminary stage of haemolysis. Indeed, the measurements of the elasticity of erythrocytes treated with triphenyltin chloride at concentrations causing less than $20 \%$ of lysis of red blood cells showed that these erythrocytes exhibited lower stiffness in contrast to intact red blood cells and cells treated with trimethyltin or tri- $n$-propyltin [10].

\section{4. ${ }^{151} E$ u as a probe of calcium binding sites in photosystem II}

Photosystem II participates in an electron transfer process extracting electrons and protons from water [25]. Photosystem II (PSII) is a protein complex located in thylakoid membranes of cyanobacteria, algae, and higher plants. Heterodimer D1/D2 is a core of photosystem II. Recent studies gave evidence that three extrinsic polypeptides $33 \mathrm{kDa}, 24 \mathrm{kDa}$, and $17 \mathrm{kDa}$ are involved in the process of transferring electrons from water on the donor side of PSII [26]. It is accepted that $\mathrm{Mn}, \mathrm{Cl}$, and $\mathrm{Ca}$ ions are necessary for the sequential four-electron water oxidation leading to molecular oxygen [5]. The role of the Mn complex is defined but this of $\mathrm{Cl}^{-}$and $\mathrm{Ca}^{2+}$ is not understood yet.

Lanthanides are good probes in the studies of $\mathrm{Ca}^{2+}$ binding sites because of the similar ionic radius and the same coordination number of the chemical 


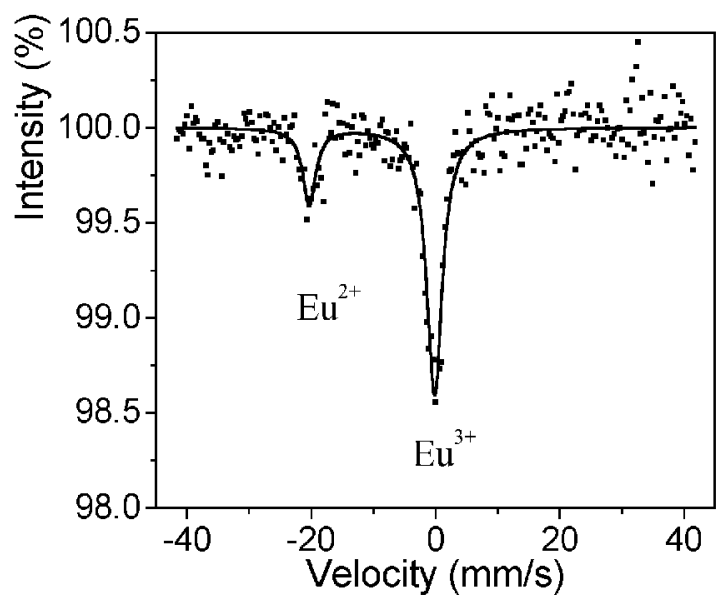

Fig. 6. ${ }^{151}$ Eu Mössbauer spectrum of PSII BBY membranes after light treatment. Note the $\mathrm{Eu}^{2+}$ and $\mathrm{Eu}^{3+}$ absorption lines.

bonds. It has been known that lanthanides destroy PSII activity and perturb the manganese complex [27]. However, we have shown that Eu and Dy can stimulate oxygen evolution when they are applied at an equimolar ratio Eu(Dy) per reaction center of PSII [13]. We found two binding sites of Eu and Dy within photosystem II. One binding site with a low affinity results in stimulation of the water splitting process, whereas that one with a high affinity leads to the inhibition of the process $[13,28]$. Our preliminary Mössbauer studies of the tightly bound $\mathrm{Eu}^{3+}$ suggest that europium (and perhaps calcium) can directly participate in the process of oxygen evolution through the binding water molecules. The Mössbauer spectrum of dark adapted PSII BBY ${ }^{\dagger}$ membranes substituted with europium ions consists only of one component corresponding to $\mathrm{Eu}^{3+}$, whereas after light treatment an additional component related to $\mathrm{Eu}^{2+}$ appears (Fig. 6). It could be the first evidence of the $\mathrm{Ca}^{2+}$ role in the photosynthetic water splitting process.

\section{Conclusions}

The aim of this paper was to present, in a rather consist way, possible applications of Mössbauer spectroscopy in studies of some problems related to chemical bonds, electrons and ions transport, and dynamics of biological systems from molecular to cellular scale. We hope that we managed to show that if other isotopes (beyond ${ }^{57} \mathrm{Fe}$ ) are applied, this local nuclear method is able to contribute in monitoring many important processes of life.

${ }^{\dagger}$ PSII BBY means photosystem II membranes prepared according to the method described in [12] by Berthold-Babcock-Yocum. 


\section{Acknowledgment}

This work was supported by grant No 6 P04A 03817 from the State Committee for Scientific Research.

\section{References}

[1] K. Spartallan, G. Lang, Oxygen Transport and Storage Materials, in: Applications of Mössbauer Spectroscopy, Ed. R.L. Cohen, Vol. II, Academic Press, New York 1980 , p. 249.

[2] R.P. Ambler, M. Daniel, J. Hermoso, T.E. Meyer, R.G. Bartsch, M.D. Kamen, Nature 278, 659 (1979).

[3] K. Strzałka, W. Subczyński, Photochem. Photobiophys. 2, 227 (1981).

[4] S.C. Liu, L.H. Derick, M.A. Duquette, J. Palek, Eur. J. Cell. Biol. 49, 358 (1989).

[5] P.H. Homann, J. Bienerg. Biomem. 19, 105 (1987).

[6] J.M. Vanderkooi, Eu. J. Biochem. 64, 381 (1976).

[7] B.F. Burnham, J.J. Zuckerman, J. Am. Chem. Soc. 92, 1547 (1970).

[8] K. Burda, Ph.D. Thesis, Niewodniczanski Inst. Nucl. Phys., Cracow 1993.

[9] K. Burda, K. Strzałka, J. Stanek, Hyp. Int. 77, 83 (1993).

[10] K. Burda, J. Lekki, J. Cieslak, J. Kruk, M. Lekka, S. Dubiel, J. Stanek, Z. Stachura, Appl. Organometal. Chem. 16, 148 (2002).

[11] M. Lekka, P. Laidler, D. Gil, J. Lekki, Z. Stachura, A. Hrynkiewicz, Eur. Biophys. J. 28, 312 (1999).

[12] D.A. Berthold, G.T. Babcock, C.F. Yocum, FEBS Lett. 134, 231 (1981).

[13] K. Burda, K. Strzałka, G.H. Schmid, Z. Naturforsch. C 50, 220 (1995).

[14] G.H. Schmid, P. Thibault, Z. Naturforsch. C 34, 414 (1979).

[15] N.N. Greenwood, T.C. Gibb, Mössbauer Spectroscopy, Chapman and Hall, London 1971.

[16] K. Burda, A. Hrynkiewicz, H. Koloczek, J. Stanek, K. Strzałka, Biochim. Biophys. Acta. 1244, 345 (1995).

[17] H. Frauenfelder, G.A. Petsko, T. Tsernoglou, Nature 280, 558 (1979).

[18] E.R. Bauminger, I. Nowik, The Dynamics of Nuclei Studied by Mössbauer Spectroscopy, in: Mössbauer Spectroscopy, Eds. D.P. Dickson, F.S. Berry, Cambridge University Press, Cambridge 1986, p. 219.

[19] H. Keller, P.G. Debrunner, Phys. Rev. Lett. 45, 68 (1980).

[20] K. Burda, A. Hrynkiewicz, H. Koloczek, J. Stanek, K. Strzałka, Hyp. Int. 91, 891 (1994).

[21] J.S. Thayer, J. Organometal. Chem. 76, 265 (1974).

[22] K.M. Attar, Appl. Organometal. Chem. 10, 317 (1996).

[23] B.M. Elliot, W.N. Aldrige, J.W. Bridges, Biochem. J. 177, 461 (1979).

[24] K.R. Siebenlist, F. Taketa, 233, 471 (1986). 
[25] G.T. Babcock, The Photosynthetic Oxygen-Evolving Process, in: New Comprehensive Biochemistry, Photosynthesis, Ed. J. Amesz, Elsevier, Amsterdam 1987, p. 125 .

[26] D.F. Ghanotakis, J.N. Topper, G.T. Babcock, C.F. Yocum, FEBS Lett. 170, 169 (1984).

[27] D.F. Ghanotakis, G.T. Babcock, C.F. Yocum, Biochim. Biophys. Acta 809, 173 (1985).

[28] K. Burda, J. Kruk, K. Strzałka, G.H. Schmid, in: PS2001 Proc. 12th Intern. Congress on Photosynthesis, Eds. K. Ahrling, R. Peace, CSIRO Publishing, Collingwood (Australia) 2001, S10-008. 